\title{
Effective interaction potentials for model amphiphilic surfactants adsorbed at fluid- fluid interfaces
}

Article

Accepted Version

Moghimikheirabadi, A., Sagis, L. M. and Ilg, P. (2018) Effective interaction potentials for model amphiphilic surfactants adsorbed at fluid-fluid interfaces. Physical Chemistry Chemical Physics, 20. pp. 16238-16246. ISSN 1463-9076 doi: https://doi.org/10.1039/C8CP01632A Available at https://centaur.reading.ac.uk/77395/

It is advisable to refer to the publisher's version if you intend to cite from the work. See Guidance on citing.

To link to this article DOI: http://dx.doi.org/10.1039/C8CP01632A

Publisher: Royal Society of Chemistry

All outputs in CentAUR are protected by Intellectual Property Rights law, including copyright law. Copyright and IPR is retained by the creators or other copyright holders. Terms and conditions for use of this material are defined in the End User Agreement.

www.reading.ac.uk/centaur 
Central Archive at the University of Reading

Reading's research outputs online 


\title{
Journal Name
}

\section{ARTICLE TYPE}

Cite this: DOI: $10.1039 / x x x x x x x x x x$

\section{Effective Interaction Potentials for Model Amphiphilic Surfactants Adsorbed at Fluid-Fluid Interfaces ${ }^{\dagger}$}

\author{
Ahmad Moghimikheirabadi, ${ }^{* a}$ Leonard M. Sagis, ${ }^{a b}$ and Patrick $\|^{c}{ }^{c}$
}

Received Date

Accepted Date

DOI: $10.1039 / x x x x x x x x x x$

www.rsc.org/journalname

\begin{abstract}
Computer simulations are a useful tool to explore the effects of interactions and structure of surfactants on interfacial microstructure and properties. Starting with "molecular-level", threedimensional reference systems of short amphiphilic surfactants at fluid-fluid interfaces, we here derive effective interaction potentials for the corresponding two-dimensional systems of structureless particles confined to the interface plane. These reference systems are comprised of two immiscible mono atomic fluids (water- and oil-like particles) and nonionic linear amphiphilic surfactants. Our results show that coarse grained interaction potentials are only weakly dependent on surface concentration but their behavior is strongly dependent on surfactant interactions. The coarse grained system preserves the in-plane surfactant center-of-mass pair correlation function at the interface and the results of surface pressure-area isotherms are in a good agreement. This approach can be extended straightforwardly to other types of surfactants at both fluid-fluid and fluid-gas interfaces providing us with an effective pairwise interaction potential for the surfactant monolayer. These effective interactions can be used to explore large-scale self-assembly within the monolayer especially at low surface concentrations where reference simulations are extremely time-consuming.
\end{abstract}

\section{Introduction}

Amphiphilic surfactants are ubiquitous in nature and industry, with a broad range of applications. They can be found in the human body (e.g. lung surfactants) 112 , are used to stabilize drug delivery systems ${ }^{3}$, are added as stabilizers in emulsions and foams in food products ${ }^{4}$, are applied in tertiary oil recovery ${ }^{5}$, and are used in nanotechnology to prepare nanostructures ${ }^{6}$. These molecules consist of both hydrophobic and hydrophilic moieties which are commonly referred to as "tail" and "head" groups respectively 7 . Due to their amphiphilic nature, they strongly adsorb at fluid-fluid (or fluid-gas) interfaces and reduce the interfacial (or surface) tension 819 . The degree of hydrophobicity (and/or hydrophilicity) of tail (and/or head) group determines the solubility of the monolayer formed at fluid-gas (or fluidfluid) interfaces ${ }^{7}$. Two-dimensional monolayers of surfactants have been subject to numerous experimental 710 - 14 , theoreti$\mathrm{cal}^{7 / 11 / 15 \mid 22}$ and molecular-level simulation studies $8[9|15| 17|23| 32$,

\footnotetext{
a Polymer Physics, Department of Materials, ETH Zürich, CH-8093 Zürich, Switzerland. Fax:+41 4463210 76; Tel: +41 4463392 32; E-mail: ahmad.moghimikheirabadi@mat.ethz.ch

${ }^{b}$ Food Physics Group, Wageningen University, Bornse Weilanden 9, 6708 WG Wageningen, The Netherlands.

${ }^{c}$ School of Mathematical, Physical and Computational Sciences, University of Reading, Reading, RG6 6AX, UK.

$\dagger$ Electronic Supplementary Information (ESI) available: See DOI: 10.1039/xxxxxxx
}

at both fluid-gas (i.e. Langmuir monolayers) and to a lesser extent at fluid-fluid interfaces.

On the experimental side, Langmuir troughs have been used widely to explore spatial and structural phase behavior of surfactant monolayers through investigating surface pressure-area isotherms $s^{7111}$. Fluorescence microscopy ${ }^{14}$, Brewster angle microscopy 33 and $\mathrm{x}$-ray surface scattering have been used to study molecular ordering and phase behavior of surfactants at both fluid-fluid and fluid-gas interfaces 7 111-13. These experimental techniques, allow us to study both spatially homogeneous and inhomogeneous phases consisting of liquid, solid or gas regions $\frac{12}{12}$ as well as the formation of micrometer size domains $\frac{14 \mid 33}{13}$ in surfactant monolayers. These surface features are strongly dependent on the surfactant architecture ${ }^{34}$ and even on the solvent type $\mathrm{e}^{12}$.

On the theoretical side, equations of state, such as the Volmer equation, have been used with some success to describe the pressure-area isotherms of surfactant stabilized interfaces 18 22. Empirical parameters of these models at each temperature must be determined through fitting to the experimental surface pressure-area isotherms. There are many different types of surfactants with a variety of architectures, and a behavior which is strongly affected by solvent conditions and temperature, which makes it time consuming and difficult (specially in the case of fluid-fluid interfaces) to investigate their properties through ex- 
perimental techniques. On the other hand, all atomistic simulations of Langmuir monolayers are limited to relatively small system sizes, short time scales and very fast deformation rates (in case of nonequilibrium simulations) due to the huge computational costs with available computational resources 32 . Coarse grained (CG) simulations 8 -9|15|17,23||31 , have resolved the issue of time and length scales to some extent and have provided us with useful information on the effects of surfactant architectures on interfacial properties ${ }^{25 / 35}$. But we believe these models can still be further simplified and at the same time, can preserve some important features of their reference atomistic systems, resulting in probing larger length and time scales with less computational and technical effort. Systematic approaches to dynamics of coarse-grained systems have been put forward and can be used to investigate dynamical properties of CG systems for larger time-scales 36 . Here we focus our attention to static coarsegraining and hence the length-scale bridging. Therefore, one can simulate larger systems and determine phase coexistence and domain formation which cannot be studied in all atomistic simulations of small systems as they correspond to thermodynamically metastable states 30 . Although potentials of mean force for surfactants perpendicular to the interface have already been studied 26 , to the best of our knowledge, so far there has been no attempt to derive effective interaction potentials for the surfactants (which is the lateral interaction potential between surfactant center-ofmasses) within the interfaces. To this end, we propose a mapping, from a three-dimensional (3D) molecular-level reference system (similar to the models used in Ref. $\frac{8926}{}$ ) to a 2D surfactant monolayer of structureless particles. We derive effective interaction potentials (suitable for 2D simulation of surfactant monolayers) from reference trajectories, by using a multiscale-coarse graining (MS-CG) scheme 4041 . The effects of solvents, internal structure and underlying molecular-level interaction potentials are all incorporated into this effective pairwise potential. This mapping procedure can be extended straightforwardly to other types of surfactants both at fluid-fluid and fluid-gas interfaces, resulting in an effective 2D fluid, computationally far more efficient and simpler than their reference molecular-level systems. More importantly, given an effective pairwise interaction potential, one can explore the spatial (not the structural) phase behavior of Langmuir monolayer of short surfactants (which corresponds to gaseous, liquid phases and their coexistence regions ${ }^{7}$ ) by using e.g. well-established liquid state theories 42 . This is especially important for the gaseous-liquid expanded phase transition of Langmuir monolayers which happens at very low surface concentrations $^{7}$ and is computationally expensive to explore in a full 3D atomistic simulation. A very large 3D system is needed to accommodate enough surfactants at the interface such that one can reduce finite-size effects and perform a reasonable statistical analysis. We verify the strong dependency of the surface features in Langmuir monolayers on surfactant structure ${ }^{34}$ via comparing effective interaction potentials between two very similar reference systems for the same surfactant architecture $\mathrm{H}_{5} \mathrm{~T}_{5}$ with slightly different molecular-level interactions, which we call "symmetric" and "asymmetric" cases throughout this work. We also explore the effect of surface concentration on the effective interaction po- tentials for both cases.

The remainder of this manuscript is organized as follows. In the next section, details about the molecular-level reference model simulations, mapping operator and consistency conditions of MSCG are explained. Supporting details regarding local pressure tensor calculations and the force matching scheme are discussed in the Supplementary Information. Results of the MS-CG for two different sets of molecular-level interactions at several surface concentrations, and Monte Carlo (MC) simulations of corresponding 2D systems are discussed in the Results section. Finally, we summarize our findings in the Conclusions.

\section{Multiscale coarse graining}

In this section, we begin by defining the molecular-level reference system that has been introduced in Ref. 9 (with a different surfactant architecture) to model linear, flexible nonionic amphiphilic surfactants dispersed in oil- and water-like fluids. Corresponding simulation results of pressure and density profiles are shown and discussed. Then, we describe how the coarse graining scheme is applied to these systems in order to derive effective interaction potentials.

\subsection{Reference simulations}

We choose a simple well-known molecular model that has been studied in the literature 9 as our reference ternary system. The reference system consists of two immiscible fluid particles namely $\mathrm{o}$ and $\mathrm{w}$ (oil-like and water-like particles) and linear amphiphilic surfactant chains consisting of five head and five tail beads, $\mathrm{H}_{5} \mathrm{~T}_{5}$. A conventional bead-spring model for the chain molecules and truncated and shifted Lennard-Jones (LJ) potential for all nonbonded interaction pairs within the system $\frac{8[9] 25[26] 35 \mid 43}{4}$ are used

$u_{i j}(r)= \begin{cases}4 \varepsilon_{i j}\left(\left(\frac{\sigma_{i j}}{r}\right)^{12}-\left(\frac{\sigma_{i j}}{r}\right)^{6}\right)-4 \varepsilon_{i j}\left(\left(\frac{\sigma_{i j}}{r_{\mathrm{cut}, i j}}\right)^{12}-\left(\frac{\sigma_{i j}}{r_{\mathrm{cut}, i j}}\right)^{6}\right), & r \leqslant r_{\mathrm{cut}, i j} \\ 0, & r>r_{\mathrm{cut}, i j}\end{cases}$

where $\varepsilon_{i j}$ is the potential well depth and $\sigma_{i j}$ is the average atomic diameter of particles $i$ and $j$. Equation (1) represents the nonbonded interaction potential between a single pair of particle types $i$ and $j$ ( $i$ and $j$ runs over all species i.e. o, w, H, T), the sum of which over all pairs of particles within the system gives us the system non-bonded potential energy. Identical particles as well as water-head (w-H) and oil-tail (o-T) interact via the full $\mathrm{LJ}$ potential and thus prefer to reside in close neighborhood, while all other pairs of particles are disfavored (by setting the cut-off distance to $2^{1 / 6} \sigma$ ) to drive a (micro) phase separation.

In order to explore the effects of surfactant structure on the effective interaction potential, we have considered two sets of interaction potentials. The first set are interaction potentials for symmetric surfactants, for which all the monomers and solvent particles have the same size $\sigma_{i j}=\sigma$ and the same potential well depth $\varepsilon_{i j}=\varepsilon$. These interaction parameters are summarized in Table 1

The second set are interaction potentials for asymmetric surfactants, where the potential well depth between $\mathrm{H}$ segments is set to $\varepsilon_{\mathrm{H}-\mathrm{H}} / \varepsilon=1.2$, such that these segments have more affinity to- 
Table 1 Non-bonded interaction potential parameters, Eq. 11, for symmetric surfactants. The truncated and shifted repulsive branch of the LJ potential (i.e. Weeks-Chandler-Andersen potential) is used for $\mathrm{w}-\mathrm{O}, \mathrm{W}-\mathrm{T}$, $\mathrm{o}-\mathrm{H}, \mathrm{H}-\mathrm{T}$ pairs. All other pairs interact via shifted LJ potential with the cut-off distance of $2.5 \sigma$.

\begin{tabular}{lllll}
\hline type $i$ & type $j$ & $\varepsilon_{i j} / \varepsilon$ & $\sigma_{i j} / \sigma$ & $r_{\text {cut }, i j} / \sigma$ \\
\hline $\mathrm{W}$ & $\mathrm{w}$ & 1.0 & 1.0 & 2.5 \\
$\mathrm{w}$ & $\mathrm{o}$ & 1.0 & 1.0 & $2^{1 / 6}$ \\
$\mathrm{w}$ & $\mathrm{H}$ & 1.0 & 1.0 & 2.5 \\
$\mathrm{w}$ & $\mathrm{T}$ & 1.0 & 1.0 & $2^{1 / 6}$ \\
$\mathrm{o}$ & $\mathrm{O}$ & 1.0 & 1.0 & 2.5 \\
$\mathrm{o}$ & $\mathrm{H}$ & 1.0 & 1.0 & $2^{1 / 6}$ \\
$\mathrm{o}$ & $\mathrm{T}$ & 1.0 & 1.0 & 2.5 \\
$\mathrm{H}$ & $\mathrm{H}$ & 1.0 & 1.0 & 2.5 \\
$\mathrm{H}$ & $\mathrm{T}$ & 1.0 & 1.0 & $2^{1 / 6}$ \\
$\mathrm{~T}$ & $\mathrm{~T}$ & 1.0 & 1.0 & 2.5 \\
\hline
\end{tabular}

wards each other compared to symmetric surfactants. All other interaction potential parameters including cut-off distances are the same as in the symmetric surfactant case.

The harmonic bond potential between successive beads within polymer chains was set equal to:

$$
u_{b}(r)=\frac{1}{2} k\left(r-l_{0}\right)^{2}
$$

where $k$ is the spring constant and $l_{0}$ is the equilibrium separation, which are set to $k=100 \varepsilon / \sigma^{2}$ (large enough to prevent chain crossing) and $l_{0}=1.0 \sigma$ for all cases 9 .

The open-source molecular dynamics simulation package LAMMPS 44 is used to perform all 3D simulations in the canonical ensemble. For all cases, we have used a system of 166400 particles (solvent and monomer) inside a simulation box with dimensions $L_{x}=L_{y}=50.56 \sigma, L_{z}=92.96 \sigma$ and overall particle density of $\rho=0.7 \sigma^{-3}$. Periodic boundary conditions are used in all three directions $x, y$ and $z$. The masses of all particles (solvent and monomer) are the same $(m)$ and we have used an integration time step of $0.005 \sigma \sqrt{m / \varepsilon}$. A Nosé-Hoover thermostat ${ }^{45}$ with damping time of $0.5 \sigma \sqrt{m / \varepsilon}$ is used to keep the temperature fixed at $T=1.0 \varepsilon / k_{\mathrm{B}}$. The same state point $\left(\rho=0.7 \sigma^{-3}, T=1.0 \varepsilon / k_{\mathrm{B}}\right)$ has been studied before in Ref. 9 .

Each simulation consists of two main intervals followed by a sampling interval. In the first interval, we start the simulation from an initial configuration at which one layer of o particles (83200 total) are sandwiched with respect to the $z$-direction between two layers of $\mathrm{w}$ particles (83200 total, which are connected via the periodic boundaries) in a fcc lattice (with no polymer chains). We let this system relax for 50000 time steps, while the interface is found to remain intact and oriented normal to the $z$-direction. Afterwards, for each polymer chain $\mathrm{H}_{5} \mathrm{~T}_{5}$ to be inserted, we select randomly 10 adjacent solvent particles from the interfacial region (either upper or lower interface) where the first 5 particles are of w-type and the rest are of the o-type and the distance between each pair of successively selected particles is less than $1.5 \sigma$. Subsequently, we change the identity of selected $w$ (and o) particles to $\mathrm{H}$ (and $\mathrm{T}$ ) and connect the successive particles with harmonic springs defined in Eq. (2). Depending on the total number of surfactants $N$, we continue this procedure until placing half of them at each interface. Now the system is ready for the second interval in which we continue the simulation for another $10^{5}$ time steps before starting sampling the equilibrium state. We monitor system pressure and temperature to make sure the system has equilibriated and we are sampling the equilibrium state. Data are then collected once every 20 steps for the final $10^{5}$ sampling time steps.

Figure 1 1 represents an equilibrium snapshot of a system corresponding to asymmetric surfactants, comprised of an equal number of o and w particles $\left(N_{\mathrm{o}}=N_{\mathrm{w}}=77200\right)$ and $1200 \mathrm{H}_{5} \mathrm{~T}_{5}$ surfactants where the average surface concentration is $\Gamma=\frac{N}{2 L_{x} L_{y}}=$ $0.234 \sigma^{-2}$. By visualizing the equilibrium snapshots (for differ-

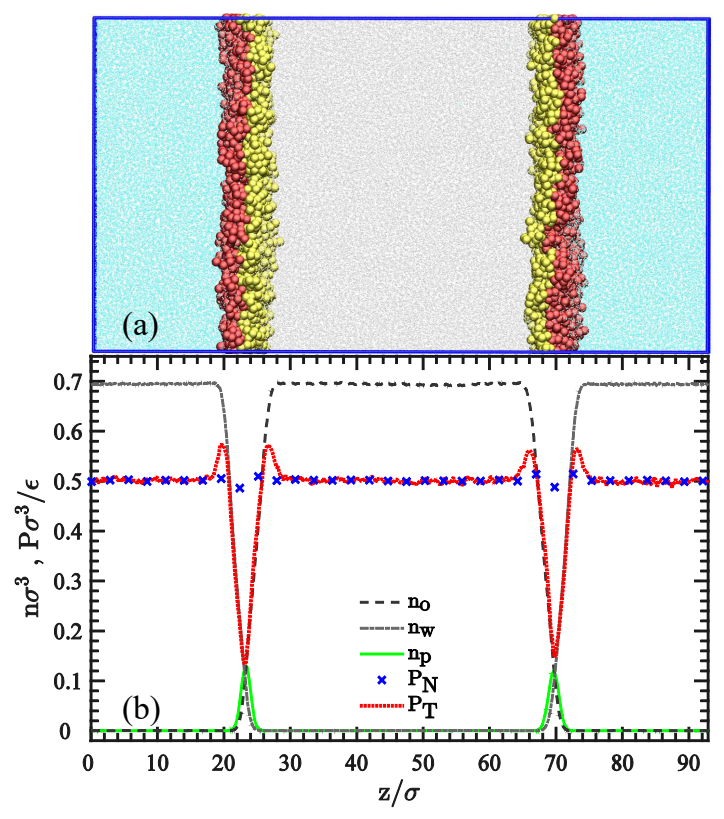

Fig. 1 (a) An equilibrium snapshot of the system with $N_{\mathrm{o}}=N_{\mathrm{w}}=77200$ and $1200 \mathrm{H}_{5} \mathrm{~T}_{5}$ asymmetric surfactants $\left(\Gamma=0.234 \sigma^{-2}\right)$. Blue (and gray) dots stand for $\mathrm{w}$ (and o) particles. Red (or yellow) spheres stand for $\mathrm{H}$ (or T) beads. (b) Number densities and molecular pressure tensor components as a function of $z$, normal to the interface. $\mathrm{n}_{\mathrm{o}}, \mathrm{n}_{\mathrm{w}}$ and $\mathrm{n}_{\mathrm{p}}$ represent $\mathrm{O}, \mathrm{w}$ and surfactant $\mathrm{COM}$ number density profiles respectively. $\mathrm{P}_{\mathrm{N}}$ and $\mathrm{P}_{\mathrm{T}}$ stand for normal and transverse components of the pressure tensor. The statistical error for $\mathrm{n}_{\mathrm{o}}, \mathrm{n}_{\mathrm{w}}, \mathrm{n}_{\mathrm{p}}$ is $0.001 \sigma^{-3}$ and for $\mathrm{P}_{\mathrm{N}}$ and $\mathrm{P}_{\mathrm{T}}$ is $0.008 \varepsilon \sigma^{-3}$ and $0.012 \varepsilon \sigma^{-3}$ respectively.

ent surfactant surface concentrations in two cases) we can observe that surfactants preferably attach to the interfaces and form rather planar monolayers. We support our observations by calculating density profiles for all species. Figure $1 \mathrm{p}$ illustrates o, w and $\mathrm{H}_{5} \mathrm{~T}_{5}$ center-of-mass (COM) number density profiles, as $n_{\mathrm{o}}$, $\mathrm{n}_{\mathrm{w}}$ and $\mathrm{n}_{\mathrm{p}}$ respectively. These profiles are symmetric with respect to the center of the simulation box and the solvent densities are quite uniform in the bulk phases. The density of surfactants is close to zero within the bulk phases and is sharply peaked at the interfacial region. We investigate the surfactant monolayer film more precisely in Figure 2, where $\mathrm{n}_{\mathrm{p}}$ as a function of distance from the center of the simulation box is plotted at different surface concentrations for both symmetric and asymmetric surfactants. By 


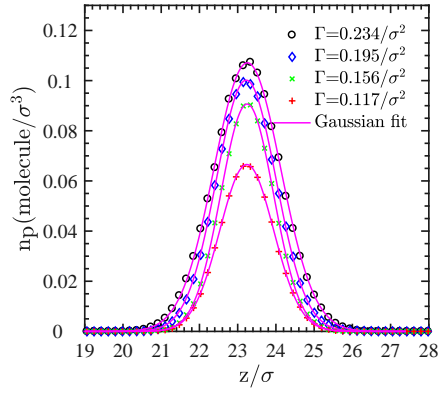

(a)

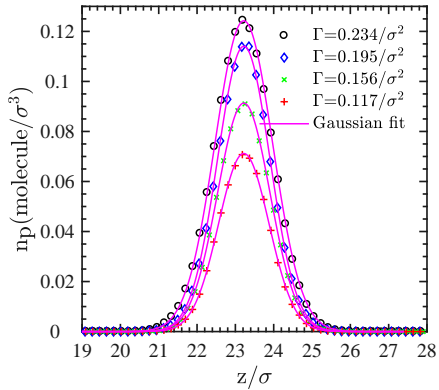

(b)

Fig. 2 Number density profiles of surfactants (based on the position of COMs) at different surface concentrations (symbols) and Gaussian fits (solid lines) for symmetric (a) and asymmetric (b) cases.

fitting Gaussian distributions to the density profiles, the width of the surfactant COM distribution in the $z$-direction for symmetric and asymmetric cases have been calculated as $4.68 \pm 0.56 \sigma$ and $4.23 \pm 0.28 \sigma$ respectively over the examined surface concentration range. Therefore, the $z$-component of the surfactant COMs are trapped in an interface layer with thickness of a few molecular diameters only, while in the $x$ - and $y$-direction there is no such limitation, hence they can sample the whole configuration space in the $x y$-plane. We further analyze the interfacial film through considering the bond orientational order parameter $S$

$$
S=\left\langle P_{2}(\cos \theta)\right\rangle=\frac{3\left\langle\cos ^{2} \theta\right\rangle-1}{2}
$$

where $P_{2}(x)$ is the second Legendre polynomial and $\theta$ is the polar angle between bonds within the surfactants and their director (i.e. the direction normal to the interface), and the brackets denote an ensemble average. Figure 3 shows $S$ for symmetric (circles) and asymmetric (squares) surfactants adsorbed at the interface as a function of surface coverage. As can be seen in this

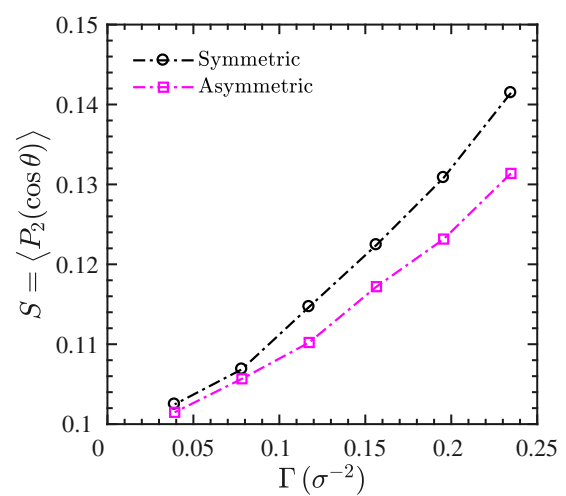

Fig. 3 Bond orientational order parameter for symmetric (circles) and asymmetric (squares) surfactants adsorbed at the interface as a function of surface coverage. The statistical error bars are smaller than the symbol sizes.

figure, there is a very weak orientational ordering even at relatively large surface concentrations for both symmetric and asymmetric cases. These arguments and observations together provide a strong motivation to integrate out the solvent particles and internal degrees of freedom of the surfactants, and develop our 3D to $2 \mathrm{D}$ mapping operator, which maps the entire 3D system to projected surfactant COMs on the $x y$-plane.

We have calculated the values of the components of the pressure tensor $\mathbf{P}$, using the Irving-Kirkwood definition 46 (see also the Supplementary Information). For interfacial systems, the condition of hydrostatic equilibrium implies that the pressure tensor is diagonal and just a function of $z$, with a constant normal component $\mathrm{P}_{\mathrm{N}}(z)$ throughout the simulation box 254647 . Considering symmetry of the system away from the interfaces, the transverse component $\mathrm{P}_{\mathrm{T}}(z)$ should be equal to $\mathrm{P}_{\mathrm{N}}(z)$ in the bulk phases. Coming back to Figure 1 , we find that $\mathrm{P}_{\mathrm{N}}$ is constant throughout the simulation box and $\mathrm{P}_{\mathrm{T}}$ is equal to $\mathrm{P}_{\mathrm{N}}$ in the bulk phases and is much reduced within the interfaces, consistent with the mechanical equilibrium condition 2546 . We have calculated the pressure tensor from both molecular 47 and atomic ${ }^{46}$ perspectives (see also the Supplementary Information), and obtained the same ensemble averages within numerical accuracy from both routes 48149 . Equality of both ensemble averages provides a further test on the successful equilibration of our configurations 50 . Numerical details about pressure tensor calculations are also given in the Supplementary Information.

\subsection{Mapping to an effective two-dimensional system}

Motivated by the fact that surfactant COMs basically remain in the interface plane, revealed by the density profiles in Figure 2 we define CG sites as projected surfactant COMs on the $x y$-plane:

$$
\mathbf{R}^{N_{\text {int }}}=\left\{\mathbf{R}_{1}, \ldots, \mathbf{R}_{N_{\text {int }}}\right\} \quad, \quad \mathbf{R}_{I} \in \mathbb{R}^{2} \quad, \quad I=1, \ldots, N_{\text {int }}
$$

where $N_{\text {int }}$ is the average number of surfactants, adsorbed at each interface plane. Over the last decades, several different coarse graining schemes have been developed for soft matter systems $37,39\left[5152\right.$. Starting with configurations $\mathbf{r}^{n}$ of the molecularlevel reference system, we follow Ref. $40 / 41$ and define the mapping operator $\mathbf{M}_{\mathbf{R}}^{N_{\text {int }}}\left(\mathbf{r}^{n}\right)=\left\{\mathbf{M}_{\mathbf{R} 1}\left(\mathbf{r}^{n}\right), \ldots, \mathbf{M}_{\mathbf{R} N_{\text {int }}}\left(\mathbf{r}^{n}\right)\right\}$ acting on the molecular-level coordinates such that $\mathbf{R}^{N_{\text {int }}}=\mathbf{M}_{\mathbf{R}}^{N_{\text {int }}}\left(\mathbf{r}^{n}\right)$. Assuming equal mass for all the beads within polymers, this operator will take the form of:

$$
\mathbf{M}_{\mathbf{R} I}\left(\mathbf{r}^{n}\right)=\frac{1}{\lambda} \sum_{j=1}^{\lambda}\left(r_{I j}^{\mathrm{p}, x} \hat{\mathbf{i}}+r_{I j}^{\mathrm{p}, y} \hat{\mathbf{j}}\right)
$$

where $\quad \mathbf{r}^{n}=\left\{\mathbf{r}_{1}^{\mathrm{w}}, \ldots, \mathbf{r}_{N_{\mathrm{w}}}^{\mathrm{w}}, \mathbf{r}_{1}^{\mathrm{o}}, \ldots, \mathbf{r}_{N_{\mathrm{o}}}^{\mathrm{o}}, \mathbf{r}_{1,1}^{\mathrm{p}}, \ldots, \mathbf{r}_{1, \lambda}^{\mathrm{p}}, \ldots, \mathbf{r}_{N_{\text {int }}, 1}^{\mathrm{p}}, \ldots, \mathbf{r}_{N_{\text {int }}, \lambda}^{\mathrm{p}}\right\}$ denotes the position vector for the molecular-level system, where $\mathbf{r}_{i}^{\mathrm{w}}, \mathbf{r}_{i}^{\mathrm{o}}$ denote positions vectors of the solvent and $\mathbf{r}_{I, j}^{\mathrm{p}}$ the position vector of the $j^{\text {th }}$ bead within the $I^{\text {th }}$ surfactant. $N_{\mathrm{w}}, N_{\mathrm{o}}$ stand for the number of $\mathrm{o}, \mathrm{w}$ particles respectively, and $\lambda$ denotes the polymerization degree of the surfactant chains. Figure 4 depicts how the mapping procedure is applied to the molecular-level reference system.

Using such a mapping operator has two advantages; first, since there are no solvent particles involved in the definition of CG sites, the resulting CG system is solvent free but their effects are incorporated implicitly in the potential of mean force for the CG sites ${ }^{40}$. Second, the system's dimension is reduced from $3 \mathrm{D}$ to $2 \mathrm{D}$, which eliminates all degrees of freedom in $z$-direction. These two 


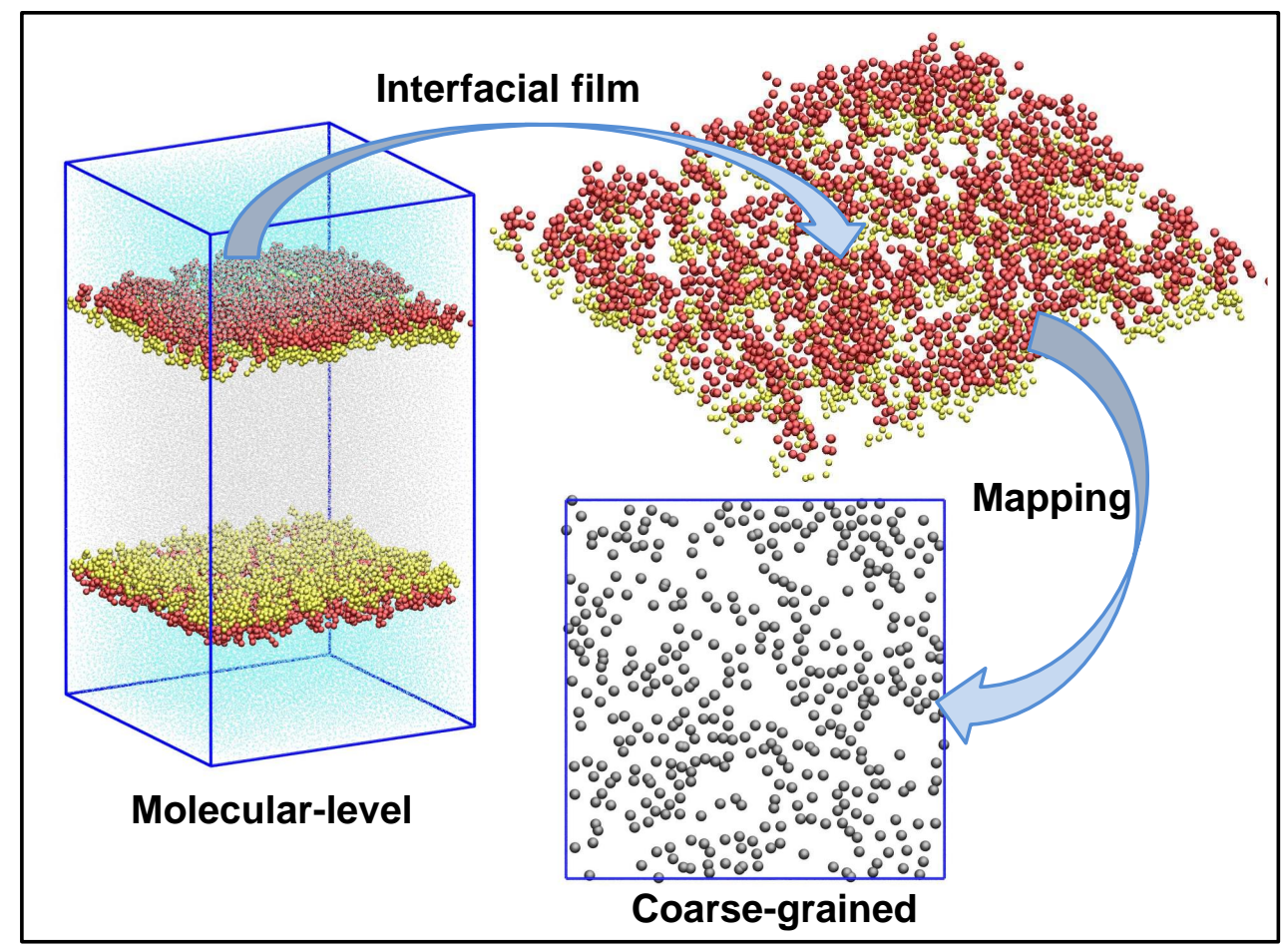

Fig. 4 Molecular-level and CG picture of the same system as Figure 1 a where the mapping operator takes the $\mathrm{H}_{5} \mathrm{~T}_{5}$ surfactant $\mathrm{COMs}$ and projects them onto the $x y$-plane. Blue and gray dots stand for $\mathrm{w}$ and o particles respectively. Red and yellow spheres stand for $\mathrm{H}$ and $\mathrm{T}$ beads respectively. Gray discs represent projected surfactants COM onto the $x y$-plane.

advantages together enable us to investigate interfacial monolayers on much larger length scales with less computational effort.

\subsection{Consistency conditions}

As alluded to above, several suggestions for defining an effective CG potential $U\left(\mathbf{R}^{N_{\text {int }}}\right)$ have been put forward in the literature. Here we follow Ref. $\frac{40}{}$ and require consistency between the coarse grained and molecular-level description of the equilibrium Boltzmann weight of the corresponding configurations :

$$
\exp \left(-\beta U\left(\mathbf{R}^{N_{\text {int }}}\right)\right) \propto \int d \mathbf{r}^{n} \exp \left(-\beta u\left(\mathbf{r}^{n}\right)\right) \prod_{I=1}^{N_{\text {int }}} \delta\left(\mathbf{M}_{\mathbf{R} I}\left(\mathbf{r}^{n}\right)-\mathbf{R}_{I}\right)
$$

where $U\left(\mathbf{R}^{N_{\text {int }}}\right)$ and $u\left(\mathbf{r}^{n}\right)$ are the CG and reference potential energy, respectively, and $\beta=1 / k_{\mathrm{B}} T$. Equation (6) implies that the CG potential energy can be determined completely (except for an additive constant) by the reference potential energy and the mapping operator. From the definition (6), the CG potential is related to the restricted canonical partition function, and should therefore be interpreted as an effective free energy which depends on density and temperature. Following the same steps as in Ref. 40 , we obtain the following relation for our coarse grained force field:

$$
\mathbf{F}_{I}\left(\mathbf{R}^{N_{\text {int }}}\right)=-\frac{\partial U\left(\mathbf{R}^{N_{\text {int }}}\right)}{\partial \mathbf{R}_{I}}=\left\langle\sum_{j=1}^{\lambda} f_{I j}^{x} \hat{\mathbf{i}}+f_{I j}^{y} \hat{\mathbf{j}}\right\rangle_{\mathbf{R}^{N_{\text {int }}}}
$$

where $\mathbf{f}_{I j}=-\frac{\partial u\left(\mathbf{r}^{n}\right)}{\partial \mathbf{r}_{I j}^{p}}$ denotes the total force exerted on the $j^{\text {th }}$ bead within the $I^{\text {th }}$ surfactant in the reference system, and the sum within brackets is the net force exerted on the $I^{\text {th }}$ surfactant COM, projected onto the $x y$-plane, which we call $\mathbf{f}_{I}$. The angular brackets represent the canonical expectation value of any continuous function $g\left(\mathbf{r}^{n}\right)$ of reference coordinates under the condition of fixed surfactant COM positions $\mathbf{M}_{\mathbf{R}}^{N_{\text {int }}}\left(\mathbf{r}^{n}\right)=\mathbf{R}^{N_{\text {int }}} 4$ :

$$
\left\langle g\left(\mathbf{r}^{n}\right)\right\rangle_{\mathbf{R}^{N_{\text {int }}}}=\frac{\int d \mathbf{r}^{n} \exp \left(-\beta u\left(\mathbf{r}^{n}\right)\right) \prod_{I=1}^{N_{\text {int }}} \delta\left(\mathbf{M}_{\mathbf{R} I}\left(\mathbf{r}^{n}\right)-\mathbf{R}_{I}\right) g\left(\mathbf{r}^{n}\right)}{\int d \mathbf{r}^{n} \exp \left(-\beta u\left(\mathbf{r}^{n}\right)\right) \prod_{I=1}^{N_{\text {int }}} \delta\left(\mathbf{M}_{\mathbf{R} I}\left(\mathbf{r}^{n}\right)-\mathbf{R}_{I}\right)}
$$

Using Equation (7) and applying a variational principle (discussed in Ref.40/41), the CG force field $\mathbf{F}$ can be obtained from underlying molecular-level simulations by minimizing the residual $\chi^{2}(\mathbf{F}) 40 \mid 41$ :

$$
\chi^{2}(\mathbf{F})=\frac{1}{2 N_{\mathrm{int}}}\left\langle\sum_{I=1}^{N_{\mathrm{int}}}\left|\mathbf{F}_{I}-\mathbf{f}_{I}\right|^{2}\right\rangle=\frac{1}{2 N_{\mathrm{int}} n_{t}} \sum_{t=1}^{n_{t}} \sum_{I=1}^{N_{\mathrm{int}}}\left|\mathbf{F}_{I, t}-\mathbf{f}_{I, t}\right|^{2}
$$

where $t$ runs over reference samples ( $n_{t}$ in total) and $\mathbf{F}_{I, t}$ (or $\mathbf{f}_{I, t}$ ) represents $\mathbf{F}_{I}$ (or $\mathbf{f}_{I}$ ) in the $t^{\text {th }}$ configuration. The present scheme and definitions automatically satisfy consistency in momentum space by assigning mass of all CG sites $M_{I}=m \lambda \lambda$.

We assume a central pairwise interaction potential $U_{\mathrm{CG}}(r) 40 \mid 4153,55$ and thus a radial pairwise forcefield $F_{\mathrm{CG}}(r)=-\frac{d U_{\mathrm{CG}}(r)}{d r}$. Therefore, the in-plane coarse grained force (left hand side of Eq. (7)) exerted on each surfactant COM can be calculated by summing all pairwise forces from neighboring surfactants in the interface plane. By using the force matching scheme which is discussed in details in the Supplementary Information, we determine the coarse grained 
forcefield $F_{\mathrm{CG}}(r)$ (and hence the coarse grained pairwise interaction potential $U_{\mathrm{CG}}(r)$ except for an additive constant) through minimization of the residual $\chi^{2}(\mathbf{F})$. Since we have considered a function space consisting of linear spline basis functions in our force matching scheme, the calculated CG forcefield is a piecewise continuous linear spline function (see Supplementary Information).

In the following section, we present the results of MS-CG at different surface concentrations and for two different sets of reference interaction potentials corresponding to symmetric and asymmetric surfactants.

\section{Results}

The results of MS-CG together with a comparison between molecular-level and CG simulations results are discussed in this section. MS-CG is performed for four different surface concentrations corresponding to $N=\{1200,1000,800,600\}$ for both symmetric and asymmetric cases. For CG simulation of systems at lower surface concentrations (e.g. $N=\{400,200\}$ ) the effective potential corresponding to $N=600$ is used.

\subsection{Symmetric surfactants}

Figure 5a depicts the results of MS-CG for the system consisting of $N=1200\left(N_{\text {int }} \approx 600\right)$ symmetric surfactants corresponding to a surface concentration of $\Gamma=0.234 \sigma^{-2}$. A common feature of

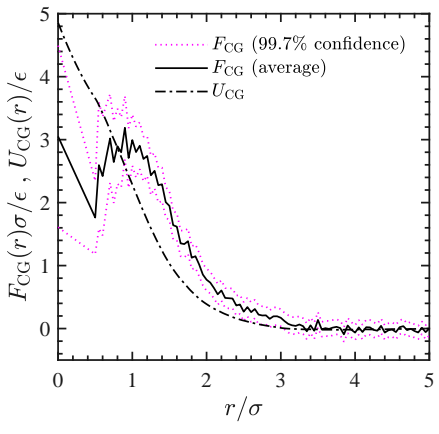

(a)

Fig. 5 (a) CG force (solid line) with its confidence intervals (dotted line) and corresponding interaction potential (dash-dotted line) for a system consisting of $1200 \mathrm{H}_{5} \mathrm{~T}_{5}$ symmetric surfactants with surface concentration of $\Gamma=0.234 \sigma^{-2}$. (b) CG interaction potentials for different surface concentrations obtained after smoothing the force data.

coarse-grained force field is that they are relatively soft. Therefore, the surfactant COMs are mutually repulsive but can possibly overlap. We support this argument by considering Figure 6 in which the pair correlation function of projected surfactant COMs onto the $x y$-plane is presented, and does not vanish at small intermolecular separations. Coming back to Figure 5a, the confidence interval shows that the forcefield is less accurate at small intermolecular distances than larger COM separations because in reference MD simulations, near zero intermolecular distances are less frequently sampled than the larger intermolecular distances. The effective interaction potential (which is obtained by integrating the average force field with a minus sign and shifting it such that $U_{\mathrm{CG}}\left(r_{\mathrm{cut}}\right)=0$ ) is shown in Figure 5b for different surface
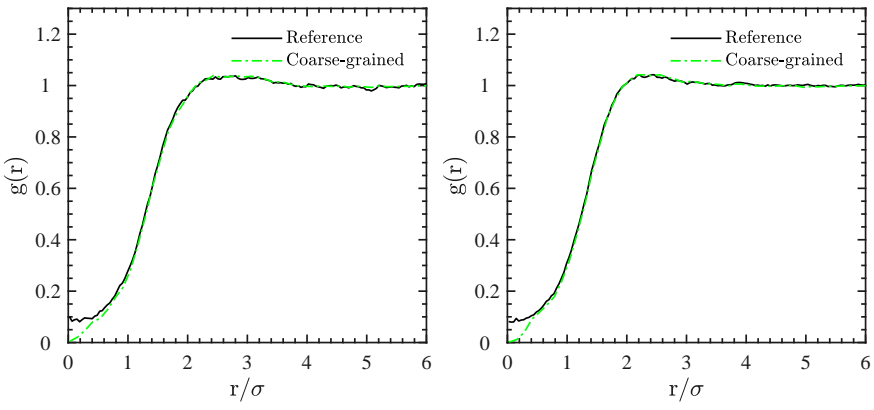

(a)

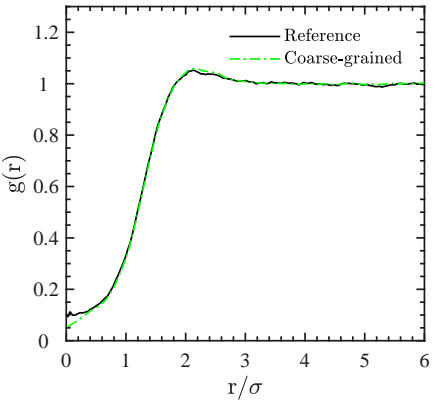

(c)

Fig. 6 Pair correlation functions of projected surfactant COMs onto the $x y$-plane for reference (solid line) and 2D CG systems (dashed line) corresponding to the symmetric case at surface concentrations of (a) $\Gamma=0.117 \sigma^{-2}$, (b) $\Gamma=0.156 \sigma^{-2}$, (c) $\Gamma=0.195 \sigma^{-2}$ and (d) $\Gamma=0.234 \sigma^{-2}$.

concentrations. Figure $5 \mathrm{~b}$ implies that the effective interaction potential does not depend strongly on the surface concentration and it gets slightly more repulsive with increasing surface concentration. Similar results for concentration-dependent interaction potentials were observed for polymer chains undergoing nonintersecting self-avoiding walks in a bulk phase ${ }^{56}$.

Using tabulated effective CG potentials, we have performed 2D MC simulations using the Metropolis scheme (as described e.g. in Ref. ${ }^{57}$ ) to validate the assumption of pairwise additivity and to check the accuracy of our force matching scheme. Figure 6 depicts a comparison between COM pair correlation functions obtained from 3D molecular-level and 2D CG simulations for different surface concentrations. The agreement is very good (which validates our force matching scheme) except for the regions with small intermolecular separation (say less than $0.5 \sigma$ ) where the matched forcefield is less accurate due to the poor sampling of such distances in the reference configurations. With increasing surface concentration the agreement gets better even at short distances due to the higher probability of sampling smaller intermolecular distances in molecular-level configurations.

In the 3D reference systems, interfacial tension can be obtained by integrating the difference between normal and transverse components of the pressure tensor in the direction normal to the interface 24 :

$$
\gamma=\frac{1}{2} \int_{-\infty}^{+\infty}\left(\mathrm{P}_{\mathrm{N}}(z)-\mathrm{P}_{\mathrm{T}}(z)\right) d z
$$

where the coefficient $\frac{1}{2}$ is due to the fact that there are two o-w interfaces in our periodic system. Using Equation (10), surface pressure can be calculated through the difference between inter- 
facial tension of the bare interface (with zero surface concentration $\Gamma=0)$ and decorated interface i.e.

$$
\Pi(\Gamma)=\gamma_{\text {bare }}-\gamma(\Gamma)
$$

In our 2D CG system, calculation of the surface pressure is simpler than in the molecular-level one, since it is nothing but the scalar pressure, $\frac{P^{x x}+P^{y y}}{2}$. The latter can be calculated from the virial theorem as

$$
\begin{aligned}
\Pi_{2 \mathrm{~d}}(\Gamma)=\Gamma k_{\mathrm{B}} T+ & \frac{1}{2 L_{x} L_{y}}\left\langle\sum_{I=1}^{N_{\text {int }}} \sum_{J>I}^{N_{\text {int }}} R_{I J} F_{\mathrm{CG}}\left(R_{I J}\right)\right\rangle \\
& +\frac{1}{L_{x} L_{y}}\left\langle\sum_{I=1}^{N_{\text {int }}} \sum_{J>I}^{N_{\text {int }}} \Gamma \frac{\partial U_{\mathrm{CG}}\left(R_{I J}\right)}{\partial \Gamma}\right\rangle .
\end{aligned}
$$

We note that Eq. 12 differs from the standard form of the virial equation by an additional term $\partial U_{\mathrm{CG}} / \partial \Gamma$ due to the densitydependence of the effective potential ${ }^{58}$. Details on the numerical evaluation of the pressure from Eq. 12 are given in the Supplementary Information. Figure 7 shows the surface pressure as a function of the average area per molecule (inverse of the surface concentration) obtained from reference and CG simulations (Equations (11) and (12) respectively). This figure shows that the CG model is quite successful in predicting surface pressure-area isotherms for the range of surface concentrations we have examined. We further investigate our MS-CG approach by considering another set of interaction potentials for our molecular-level reference system in the next section.

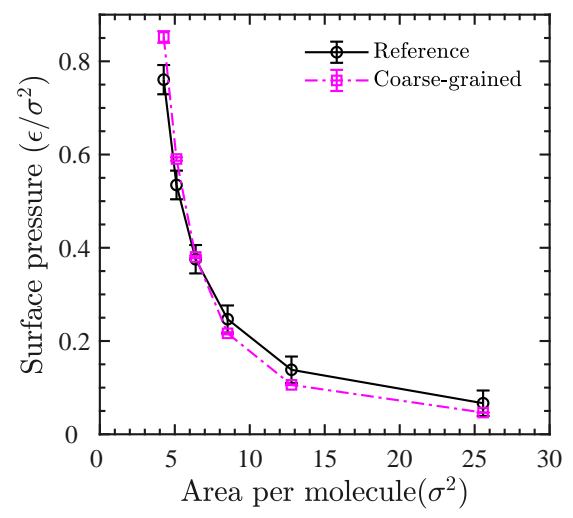

Fig. 7 Surface pressure of the molecular-level reference system (solid line) and the scalar pressure of the 2D CG system (dash-dotted line) as a function of average area per molecule (inverse of $\Gamma$ ) for the symmetric case.

\subsection{Asymmetric surfactants}

Next, we perform all our simulations and MS-CG for systems with interaction potentials corresponding to asymmetric surfactants, where the affinity between $\mathrm{H}$ types is stronger than in symmetric cases as described above. Figure 8a depicts the results of MS-CG for the system at a surface concentration of $\Gamma=0.234 \sigma^{-2}$. The CG force and effective interaction potentials are very similar to Figure 5a except the fact that the forcefield is less repulsive with respect to symmetric surfactants, and interestingly has an attrac- tive tail. This attractive tail suggests that 2D gaseous-liquid phase

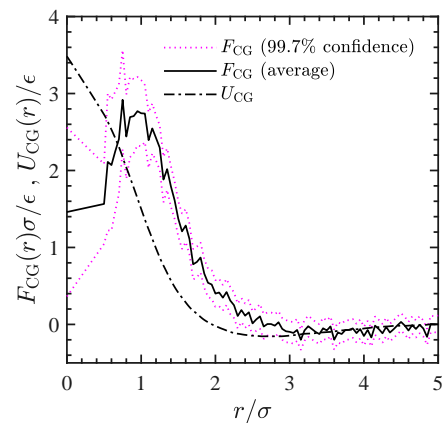

(a)

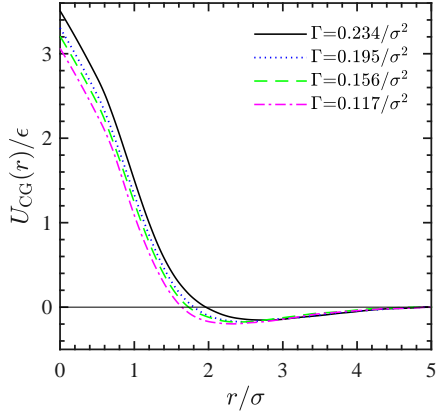

(b)
Fig. 8 (a) CG force (solid line) with its confidence intervals (dotted line) and corresponding interaction potential(dash-dotted line) for a system consisting of $1200 \mathrm{H}_{5} \mathrm{~T}_{5}$ asymmetric surfactants with surface concentration of $\Gamma=0.234 \sigma^{-2}$. (b) CG interaction potentials for different surface concentrations obtained after smoothing the force data.

transitions could occur in these interfaces (possibly at some different temperature and concentration ranges from those we have considered), similar to 2D phase transitions observed in Langmuir monolayers 7 . The force field is less accurate for small intermolecular separations again due to the lower chance of being sampled in the reference simulations, and becomes slightly more repulsive for increasing surface concentration, as is depicted in Figure $8 \mathrm{~b}$ Asymmetric surfactants are basically the same as symmetric ones but with an increased affinity between $\mathrm{H}$ blocks. This increased affinity can overcome steric repulsions for intermediate distances between molecules leading to an attractive tail in the effective interaction potential. Since the short-range effective interaction potential for higher surface concentrations is steeper (and hence more repulsive), the attractive contributions to these potentials shift the minimum to larger intermolecular distances for higher surface concentrations. A comparison between COM pair correlation functions obtained from 3D reference and 2D CG simulations for different surface concentrations is shown in Figure 9, Similar to the symmetric case, there is very good agreement between CG (dashed lines) and reference (solid lines) PCF curves, except for small molecular separations. Figures 6 and 9 further imply that the many body potential of mean force for the coarse grained system (projected surfactant COMs) can be well approximated by a sum of pairwise additive interactions $U_{\mathrm{CG}}\left(R_{I J}\right)$ between surfactant COMs.

In order to check whether our CG model can predict some characteristic features of these monolayers, we compare surface pressure-area isotherms obtained from CG (dash-dotted curve) and reference (solid curve) simulations in Figure 10. The concentration-dependence of the surface pressure in the CG system follows a similar trend as the reference one. However, deviations of the results for the CG from the reference system are more pronounced compared to the symmetric case (see Figure 7). It is well-known that CG systems cannot equally well describe structural and thermodynamic properties (such as the pressure) at the same time 59 . In the present situation, the surface pressure for the reference system is obtained via Equation (11) which also 

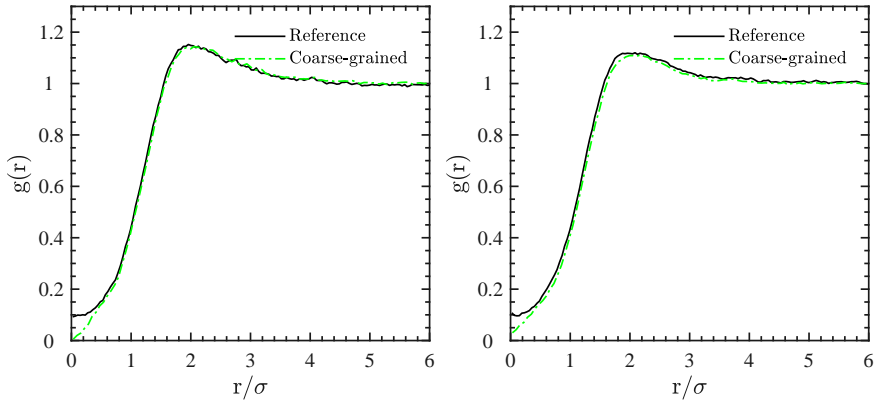

(a)

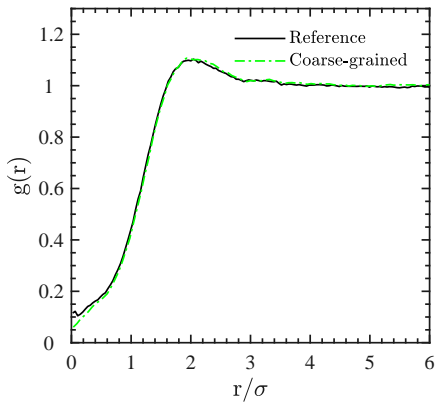

(b)

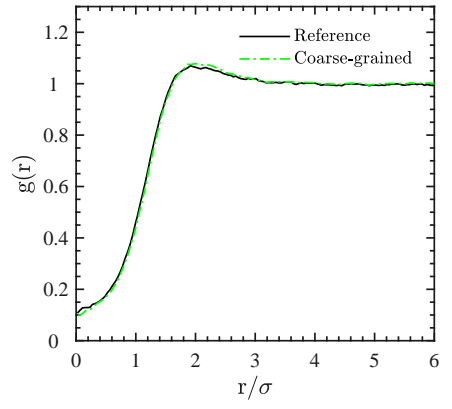

(c)

(d)

Fig. 9 Pair correlation functions of projected surfactant COMs onto the $x y$-plane for reference (solid line) and 2D CG systems (dashed line) corresponding to the asymmetric case at surface concentrations of (a) $\Gamma=0.117 \sigma^{-2}$, (b) $\Gamma=0.156 \sigma^{-2}$, (c) $\Gamma=0.195 \sigma^{-2}$ and (d) $\Gamma=0.234 \sigma^{-2}$.

involves the interfacial tension of the bare interface from which the interfacial tension of the decorated interface is subtracted. In deriving CG interaction potentials, the contribution of the bare interface is not considered, however. The interface has a finite thickness (see Figure 2) resulting in a surfactant-surfactant and surfactant-solvent contributions to the configurational part of the normal pressure $\mathrm{P}_{\mathrm{N}}$ (and hence to the surface pressure of the reference system through Equations (10) and (11)), while in the CG picture (2D interface), these contributions are missing. In view of these limitations, the agreement is satisfactory in the sense that the trends are correctly reproduced and also a semi-quantitative agreement can be reached.

\section{Conclusions}

MD simulations of molecular-level reference systems were used to derive effective coarse grained pairwise potentials for linear nonionic surfactants adsorbed at fluid-fluid interfaces via a MSCG method. The resulting 2D coarse grained representations of amphiphilic surfactants are quite reliable concerning the structure, since they can reproduce the surfactant COM pair correlation functions of the reference systems very accurately. These CG systems can also predict the surface pressure of reference monolayers semi-quantitatively. From the computational point of view, $3 \mathrm{D}$ to $2 \mathrm{D}$ mapping, together with integrating out the solvent and monomer effects, make these CG interaction potentials very efficient for large-scale simulations. One other interesting finding is that these effective interactions do not strongly depend on the surface concentration (similar to the results of Ref. 56 in coarse graining of polymer coils in bulk) which make them suitable for

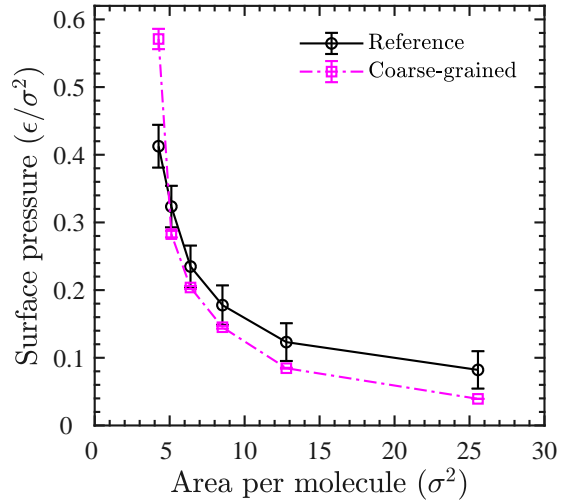

Fig. 10 Surface pressure of the molecular level reference system (solid line) and the scalar pressure of the 2D CG system (dash-dotted line) as a function of average area per molecule (inverse of $\Gamma$ ) for the asymmetric case.

investigation of the equilibrium 2D phase behavior of these monolayers.

The orientation of surfactants at the interface plane is not captured in the presented scheme but can be an important aspect of the interfacial microstructure for more rigid surfactants or higher surface concentrations. In these cases, by employing a higherlevel mapping e.g. two-site representation of surfactants (one for the COM of the head groups and another for the COM of the tail groups), the current scheme can be adapted to take into account surfactant orientational effects. Such higher level mappings can also be used to extend this work towards surfactants with more complicated chain architectures like the ones studied in Ref. ${ }^{9}$. Coarse-graining approaches often provide less reliable thermodynamic descriptions compared to structural properties. Recent developments addresses this issue within an isothermal-isobaric ensemble, and it would be interesting to apply the corresponding extended CG scheme $\frac{60}{}$ to the present case to improve the surface pressure estimation in our CG systems. CG interaction potentials obtained from MS-CG can be successful in predicting static properties. In order to capture the dynamics in the CG systems more accurately, one needs to properly account for the additional friction due to the eliminated degrees of freedom as done in systematic approaches for dynamic coarse-graining in Ref. 36. 39 .

\section{Conflicts of interest}

There are no conflicts to declare.

\section{Acknowledgements}

This work was supported by the Swiss National Science Foundation through Grant No. 200021_156106. PI acknowledges support from EU FP7-MC-CIG Grant No. 631233. The authors thank Hans Christian Öttinger and Martin Kröger for helpful discussions as well as critical reading of an earlier version of the manuscript.

\section{Notes and references}

1 J. Zasadzinski, J. Ding, H. Warriner, F. Bringezu and A. J. Waring, Curr. Opin. Coll. Surf. Sci., 2001, 6, 506 - 513.

2 S. Y. Park, R. E. Hannemann and E. I. Franses, Colloids Surf. 
$B, 1999,15,325-338$.

3 V. P. Torchilin, J. Controlled Rel., 2001, 73, 137 - 172.

4 I. Kralova and J. Sjöblom, J. Disp. Sci. Techn., 2009, 30, 13631383.

5 A. Bhardwaj and S. Hartland, J. Disp. Sci. Techn., 1993, 14, 87-116.

6 M. X. Quintanilla-Carvajal and S. Matiacevich, Food Nanoscience and Nanotechnology, Springer International Publishing, 2015, pp. 177-186.

7 V. M. Kaganer, H. Möhwald and P. Dutta, Rev. Mod. Phys., 1999, 71, 779-819.

8 A. J. Howes and C. J. Radke, Langmuir, 2007, 23, 1158011586.

9 K. Klopfer and T. Vanderlick, Colloids Surf. A, 1995, 96, 171 179.

10 K. J. Stine and D. T. Stratmann, Langmuir, 1992, 8, 25092514.

11 C. M. Knobler and R. C. Desai, Annu. Rev. Phys. Chem., 1992, 43, 207-236.

12 M. L. Schlossman and A. M. Tikhonov, Annu. Rev. Phys. Chem., 2008, 59, 153-177.

13 M. L. Schlossman, Curr. Opin. Coll. Surf. Sci., 2002, 7, 235243.

14 H. Möhwald, Thin Solid Films, 1988, 159, 1-15.

15 N. V. Os, L. Rupert, B. Smit, P. Hilbers, K. Esselink, M. Böhmer and L. Koopal, Colloids Surf. A, 1993, 81, 217-229.

16 B. D. Marshall, C. Emborsky, K. Cox and W. G. Chapman, J. Phys. Chem. B, 2012, 116, 2730-2738.

17 V. V. Ginzburg, K. Chang, P. K. Jog, A. B. Argenton and L. Rakesh, J. Phys. Chem. B, 2011, 115, 4654-4661.

18 V. B. Fainerman and R. Miller, J. Phys. Chem. B, 2000, 104, 8471-8476.

19 J. Israelachvili, Langmuir, 1994, 10, 3774-3781.

20 V. B. Fainerman and D. Vollhardt, J. Phys. Chem. B, 1999, 103, 145-150.

21 V. B. Fainerman, D. Vollhardt and V. Melzer, J. Phys. Chem., 1996, 100, 15478-15482.

22 D. Vollhardt and V. B. Fainerman, J. Phys. Chem. B, 2002, 106, 345-351.

23 S. S. Jang, S.-T. Lin, P. K. Maiti, M. Blanco, W. A. Goddard, P. Shuler and Y. Tang, J. Phys. Chem. B, 2004, 108, 1213012140.

24 A. Ghoufi, P. Malfreyt and D. J. Tildesley, Chem. Soc. Rev., 2016, 45, 1387-1409.

25 H. Popova, A. Milchev and S. A. Egorov, Colloids Surf. A, 2017, 519, $168-178$.

26 A. J. Howes and C. J. Radke, Langmuir, 2007, 23, 1835-1844.

27 S. O. Nielsen, C. F. Lopez, P. B. Moore, J. C. Shelley and M. L. Klein, J. Phys. Chem. B, 2003, 107, 13911-13917.

28 S. B. Opps, B. Yang, C. G. Gray and D. E. Sullivan, Phy. Rev. E, 2001, 63, 041602.

29 S. Karaborni and S. Toxvaerd, J. Chem. Phys., 1992, 97, 58765883.
30 S. Baoukina, L. Monticelli, S. J. Marrink and D. P. Tieleman, Langmuir, 2007, 23, 12617-12623.

31 S. Karaborni and S. Toxvaerd, J. Chem. Phys., 1992, 96, 55055515.

32 C. D. Lorenz and A. Travesset, Langmuir, 2006, 22, 10016 10024.

33 D. Vollhardt, V. B. Fainerman and S. Siegel, J. Phys. Chem. B, 2000, 104, 4115-4121.

34 D. Vollhardt and V. Fainerman, Adv. Colloid Interface Sci., 2006, 127, 83-97.

35 A. Corsi, A. Milchev, V. G. Rostiashvili and T. A. Vilgis, Food Hydrocolloids, 2007, 21, 870 - 878.

36 C. Hijon, P. Espanol, E. Vanden-Eijnden and R. DelgadoBuscalioni, Faraday Discuss., 2010, 144, 301-322.

37 P. Español, Novel Methods in Soft Matter Simulations, Springer, 2004, vol. 640, pp. 69-115.

38 P. Ilg, V. G. Mavrantzas and H. C. Öttinger, Modeling and Simulations in Polymers, Wiley-Interscience, 2010.

39 P. Ilg, H. C. Öttinger and M. Kröger, Phys. Rev. E, 2009, 79, 011802.

40 W. G. Noid, J.-W. Chu, G. S. Ayton, V. Krishna, S. Izvekov, G. A. Voth, A. Das and H. C. Andersen, J. Chem. Phys., 2008, 128, 244114.

41 W. G. Noid, P. Liu, Y. Wang, J.-W. Chu, G. S. Ayton, S. Izvekov, H. C. Andersen and G. A. Voth, J. Chem. Phys., 2008, 128, 244115.

42 J.-P. Hansen and I. R. McDonald, Theory of Simple Liquids, Academic Press, Oxford, 4th edn, 2013.

43 M. T. da Gama and K. Gubbins, Mol. Phys., 1986, 59, 227239.

44 S. Plimpton, J. Comput. Phys., 1995, 117, 1 - 19.

45 W. G. Hoover, Phys. Rev. A, 1985, 31, 1695-1697.

46 J. Walton, D. Tildesley, J. Rowlinson and J. Henderson, Mol. Phys., 1983, 48, 1357-1368.

47 J. Alejandre, D. J. Tildesley and G. A. Chapela, J. Chem. Phys., 1995, 102, 4574-4583.

48 R. L. C. Akkermans and G. Ciccotti, J. Phys. Chem. B, 2004, 108, 6866-6869.

49 D. N. Theodorou, T. D. Boone, L. R. Dodd and K. F. Mansfield, Macromol. Theory Simul., 1993, 2, 191-238.

50 J. G. Harris, J. Phys. Chem., 1992, 96, 5077-5086.

51 E. Brini, E. A. Algaer, P. Ganguly, C. Li, F. Rodríguez-Ropero and N. F. A. van der Vegt, Soft Matter, 2013, 9, 2108-2119.

52 A. R. Denton, Nanostructured Soft Matter, Springer, Netherlands, 2007, pp. 395-433.

53 S. Izvekov and G. A. Voth, J. Chem. Phys., 2005, 123, 134105.

54 S. Izvekov and G. A. Voth, J. Phys. Chem. B, 2005, 109, 24692473.

55 S. Izvekov, M. Parrinello, C. J. Burnham and G. A. Voth, J. Chem. Phys., 2004, 120, 10896-10913.

56 A. A. Louis, P. G. Bolhuis, J. P. Hansen and E. J. Meijer, Phys. Rev. Lett., 2000, 85, 2522-2525.

57 M. P. Allen and D. J. Tildesley, Computer Simulation of Liquids, Clarendon Press, New York, NY, USA, 1989. 
58 P. Ascarelli and R. J. Harrison, Phys. Rev. Lett., 1969, 22, 385388.

59 A. A. Louis, Faraday Discuss., 2010, 144, 323-345.
60 N. J. H. Dunn and W. G. Noid, J. Chem. Phys., 2015, 143, 243148. 